\title{
TIGIT-Fc Promotes Antitumour Immunity
}

$4{ }^{1}$ Department of Biophysics, College of Basic Medical Sciences, Second Military Medical

5 University, Shanghai 200433, China.

$6 \quad 2$ Department of Assisted Reproduction, Shanghai Ninth People's Hospital, Shanghai Jiao Tong

7 University School of Medicine, Shanghai 200011, China.

$8 \quad{ }^{3}$ KOCHKOR Biotech, Inc., Shanghai 202152, China

9

10 *Corresponding Author: Shi Hu, Second Military Medical University, 800 Xiangyin Road,

11 Shanghai, China, 200433. Phone:+86-02181870925. E-mail: hus@smmu.edu.cn.

12

13

14 Keywords: Cancer Immunotherapy, TIGIT, Fc fusion, $\mathbf{N K}$ cell, $\mathbf{T}$ cell 


\section{Abstract}

21 T cell immunoreceptor with Ig and ITIM domains (TIGIT) is a checkpoint receptor that

22 mediates both $\mathrm{T}$ cell and natural killer (NK) cell exhaustion in tumours. An Fc-TIGIT

23 fusion protein was shown to induce an immune-tolerance effect in a previous report, but

24 the relevance of the TIGIT-Fc protein to tumour immunity is unknown. Here, we

25 unexpectedly found that TIGIT-Fc promotes rather than suppresses tumour immunity.

26 TIGIT-Fc treatment promoted the effector function of $\mathrm{CD}^{+} \mathrm{T}$ and NK cells in several

27 tumour-bearing mouse models. Additionally, TIGIT-Fc treatment resulted in potent T cell

28 and NK-cell-mediated tumour reactivity, sustained memory-induced immunity in tumour

29 re-challenge models, enhanced therapeutic effects via an antibody against PD-L1, and

30 induction of Th1 development in $\mathrm{CD}^{+} \mathrm{T}$ cells. TIGIT-Fc showed a potent

31 antibody-dependent cell-mediated cytotoxicity (ADCC) effect but no intrinsic effect on

32 tumour cell development. Our findings elucidate the unexpected role of TIGIT-Fc in

33 tumour immune reprogramming, suggesting that TIGIT-Fc treatment alone or in

34 combination with other checkpoint receptor blockers is a promising anticancer

35 therapeutic strategy. 
T cell immunoreceptor with Ig and ITIM domains (TIGIT, also known as WUCAM,

48 Vstm3 or VSIG9), which is an inhibitory receptor expressed on lymphocytes belonging to

49 the receptor of the Ig superfamily regulatory network that involves multiple players (e.g.,

50 CD96/TACTILE, CD112R/PVRIG), is a major emerging target for cancer

51 immunotherapy ${ }^{1}$. As an inhibitor of antitumour responses, TIGIT has the capacity to

52 hinder multiple steps of the cancer immunity cycle, and therefore it is of interest in the

53 development of a first-in-class checkpoint-blocking drug ${ }^{2}$; in addition, new clinical data

54 has shown that combination therapy with the anti-TIGIT antibody tiragolumab combined

55 with atezolizumab appears safe and effective against non-small cell lung cancer

56 (NSCLC) $)^{3}$. Several mechanisms of action have been proposed for TIGIT-mediated

57 inhibition of effector $\mathrm{T}$ cells and NK cells and the suppression of tumour-specific

58 immunity. TIGIT was reported to interfere with the co-stimulation effect of DNAM- ${ }^{4}$ or

59 to directly deliver inhibitory signals to the effector cells ${ }^{5}$. Additionally, TIGIT was

60 reported to enhance the suppressive functions of regulatory T cell (Tregs) and hence have

61 the potential to inhibit a wide range of immune cells ${ }^{6,7}$.

62 TIGIT-Fc is an Fc fusion protein in which the extracellular domain of TIGIT is fused 63 genetically to the immunoglobulin Fc domain. This genetic method enables Fc fusion 64 proteins to have some antibody-like properties, such as long serum half-life and easy 65 expression and purification, making them an attractive platform for research agents and 66 therapeutic drugs ${ }^{8}$. In a previous report, TIGIT-Fc showed an immunosuppressive effect

67 by inhibiting $\mathrm{T}$ cell activation in vitro in a dendritic cell-dependent manner and inhibited 68 delayed-type hypersensitivity reactions in mice ${ }^{9}$. We previously also showed that 69 TIGIT-Fc demonstrates a therapeutic effect in a mouse model of lupus ${ }^{10}$. Interestingly, 70 TIGIT-Fc was also shown to promote NK cell activation ${ }^{11}$ and had no effect on cytokine 71 production by tumour-specific $\mathrm{CD}^{+} \mathrm{T}$ cells in vitro ${ }^{12}$, indicating that TIGIT-Fc may be a 72 multifaceted immunomodulator and that its function may be dependent on the immune 73 microenvironment. In particular, the impact of TIGIT-Fc on antitumour immunity is 74 currently unknown.

75 Here, we unexpectedly found that TIGIT-Fc notably reduced the growth of human 
76 tumours in a xenograft model containing coimplanted human $\mathrm{T}$ cells by supporting a 77 stimulatory immunological mechanism of action for TIGIT-Fc. TIGIT-Fc treatment alone

78 was sufficient to delay tumour growth in vivo and reverse the exhaustion of antitumour $\mathrm{T}$ 79 cells and NK cells in multiple tumour models. We further demonstrated that TIGIT-Fc not 80 only directly subverted the exhaustion of tumour-infiltrating NK cells, similar to the 81 effect of blockade of TIGIT with antibodies, but also sustained tumour-specific $\mathrm{T}$ cell 82 function in a $\mathrm{CD}^{+} \mathrm{T}$ cell-dependent manner. Moreover, a synergistic effect was achieved 83 by the combined therapy of TIGIT-Fc and PD-L1 blockade. TIGIT-Fc also potentially 84 mediated the ADCC lysis of tumour cells in vitro. Hence, these findings demonstrate that 85 TIGIT-Fc may coordinate both the reversal of NK cell exhaustion and the acceleration of 86 the activation of $\mathrm{T}$ cells for tumour control and support the clinical development of 87 TIGIT-Fc for cancer immunotherapy. 


\section{Results}

\section{TIGIT-Fc unexpectedly shows antitumour immunity}

106 Targeting co-inhibitory receptors is highly relevant in cancer where therapeutic 107 effects are being exploited clinically. PD-1/PD-L1 blockers are efficacious in the 108 treatment of cancer, but resistance to this therapy is increasing, and the responses to 109 anti-PD-1 or anti-CTLA-4 immunotherapy are relatively minimal in some tumours (such

110 as colorectal carcinomas). Recent reports have shown that anti-TIGIT antibodies have 111 synergistic effects with PD-1 blockade in cancer, both in pre-clinical and clinical studies. 112 As we and others previously have shown that TIGIT-Fc is an immune-suppressive 113 agents $^{9,10}$, we initially aimed to test whether TIGIT-Fc has the capacity to promote 114 resistance to anti-PD-L1 therapy in xenograft models of the human melanoma cell line 115 A375. Both TIGIT-Fc and atezolizumab were confirmed to bind to A375 cells (Fig. 1a). 116 To generate allogenic T cell lines with specificity to A375 cells ${ }^{13}$, primary human T cells 117 were expanded in culture and implanted subcutaneously in NSG mice together with 118 tumour cells. Our data shows that the administration of the anti-PD-L1 antibody 119 atezolizumab led to a notable growth inhibition of the A375 tumour in the presence of 120 human $\mathrm{T}$ cells, consistent with a previous report ${ }^{13}$. Unexpectedly, TIGIT-Fc alone 121 significantly inhibited the tumour growth of A375 xenografts compared with an 122 isotype-matched control antibody. Moreover, the combined therapy of TIGIT-Fc and 123 atezolizumab therapy showed a superior tumour growth inhibition reaching nearly 100\% 124 (Fig. 1b). Based on the immunoglobulin-like structure of TIGIT-Fc, we hypothesize that 125 TIGIT-Fc may have a two-sided biological effect in that it is a potent agonist of CD155 126 and a membrane-bound TIGIT antagonist. We tested the antitumour effect of the 127 anti-TIGIT antibody etigilimab in the T cell-based A375 tumour model, but etigilimab 128 alone showed no inhibitory effect on the growth of the A375 tumour xenograft, and 129 combined therapy with etigilimab and atezolizumab showed no synergistic effect 130 compared with atezolizumab monotherapy (Fig. 1c), suggesting that the antitumour 131 efficacy of TIGIT-Fc is not due to TIGIT blockade in A375 xenografts.

\section{TIGIT-Fc enhances effector NK cell function in tumour-bearing mice}

133 A previous report showed that blockade of TIGIT prevents NK cell exhaustion ${ }^{14}$. To 
134 assess whether TIGIT-Fc can blockade TIGIT on NK cells similar to that of an 135 anti-TIGIT antibody in tumour-bearing mice, murine TIGIT-Fc (mTIGIT-Fc) was first 136 tested against subcutaneous MC38 colon adenocarcinoma tumours, since this model is 137 considered a standard and has been demonstrated by many laboratories as 138 anti-PDL1-sensitive and immunogenic.

139 In the MC38 tumour model (Fig. 2a), we found that tumour growth was notably 140 inhibited by the administration of mTIGIT-Fc, as shown by the lower tumour volume (Fig. $1412 \mathrm{a}$ ) and the improved overall survival of mice (Fig. 2b). Moreover, three of eight mice 142 with the mTIGIT-Fc treatment showed complete tumour regression, suggesting that 143 TIGIT-Fc has the capacity to induce a strong antitumour immunity. Administration of

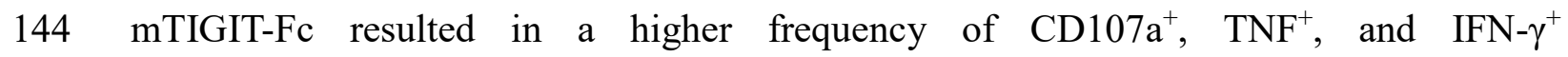
145 tumour-infiltrating NK cells than in mice treated with the control IgG (Fig. 2c), indicating 146 that mTIGIT-Fc reversed the exhaustion of the tumour-infiltrating NK cells. Of note, a 147 significantly higher frequency of tumour-infiltrating $\mathrm{CD} 8^{+} \mathrm{T}$ cells with surface expression 148 of CD107a, TNF and IFN- $\gamma$ was also observed to accompany the mTIGIT-Fc treatment. 149 Another colorectal carcinoma mouse model, CT26, was also employed in our study, in 150 which it was observed that mTIGIT-Fc delayed tumour growth and prolonged overall 151 mouse survival (Fig. 2e-f), and two of eight mice showed complete tumour regression 152 with the mTIGIT-Fc treatment. Consistent with the observation in MC38 tumours, 153 mTIGIT-Fc alleviated NK cell exhaustion, as a higher frequency of tumour-infiltrating 154 NK cells expressing CD107a, TNF and IFN- $\gamma$ were observed. Additionally, mTIGIT-Fc 155 treatment showed a higher frequency of tumour-infiltrating $\mathrm{CD} 8^{+} \mathrm{T}$ cells expressing TNF 156 and IFN- $\gamma$. Thus, these results indicated that TIGIT-Fc shows a similar TIGIT blockade

157 effect as an anti-TIGIT antibody to prevent the exhaustion of NK cells in tumour-bearing 158 mice with a beneficial effect on T cells.

159 TIGIT-Fc has a strong antitumour effect on CD155-positive tumours in the absence 160 of adaptive immunity.

161 We then determined whether the antitumour effect of TIGIT-Fc can occur in nude 162 mice that lack host T cells. In CT26 tumour model based on nude mice, we found that 163 following treatment with mTIGIT-Fc, CT26 tumour growth was notably inhibited (Fig. $1643 \mathrm{a}$ ), and the tumour-infiltrating NK cells showed improved function, as indicated by the 
165 increased frequency of such cells expressing CD107a, TNF or IFN- $\gamma$ in the nude mice 166 model (Fig. 3b). The therapeutic value of the recombinant human TIGIT-Fc-targeting 167 regimen was further assessed in mice xenografted with human cancer cells. As shown in 168 Fig. 4c, the vehicle A431 tumours progressed rapidly and reached volumes of greater than $1691,000 \mathrm{~mm}^{3}$ in less than 30 days. Conversely, TIGIT-Fc treatment effectively delayed tumour growth to this volume for approximately 40 days. TIGIT-Fc treatments also efficiently inhibited tumour growth and delayed tumour growth for 45 days and 30 days in SK-BR-3 or SK-OV-3 tumours, respectively (Fig. 3c). As we detected high CD155

173 expression in the mouse tumour cell line CT26 and in the human tumour cell line A431, 174 SK-BR-3 cells and SK-OV-3 cells (Fig. 4d), we therefore use H2126 cells with very low 175 detectable CD155 expression to further evaluate the antitumour role of TIGIT-Fc.

Interestingly, we observed that the therapeutic benefit of TIGIT-Fc treatment on tumour growth in the CD155 low-expression model was minimal (Fig. 4c). The CD155 178 expression of tumour cells contributing to the antitumour effect of TIGIT-Fc was further 179 supported in the tumour model of H2126 cell derivatives engineered to express CD155, 180 in which TIGIT-Fc showed a notable antitumour effect in tumour-bearing mice. In all the 181 tumour models based on nude mice, despite the delayed tumour growth and survival 182 advantage, mice treated with TIGIT-Fc eventually succumbed to the tumours, and no 183 mice had the capacity to reject tumour cells, suggesting that the antitumour effect of 184 TIGIT-Fc would be weakened by dysfunction of the host $\mathrm{T}$ cells.

\section{$185 \mathbf{C D 4}^{+} \mathbf{T}$ cells have a pivotal role in the antitumour immunity of TIGIT-Fc}

186 To further explore the mechanism that underlies the antitumour immunity of 187 TIGIT-Fc, mice were depleted of $\mathrm{NK}$ cells, $\mathrm{CD}^{+} \mathrm{T}$ cells, and $\mathrm{CD}^{+} \mathrm{T}$ cells in 188 combination with mTIGIT-Fc treatment in syngeneic immune-competent mouse models. 189 In CT26 tumour models, we found that the depletion of $\mathrm{CD}^{+} \mathrm{T}$ cells, $\mathrm{CD}^{+} \mathrm{T}$ cells or 190 NK cells significant inhibited the therapeutic effect of mTIGIT-Fc (Fig. 4a-b). A previous 191 report showed that depletion of NK cells abolished the therapeutic effect of anti-TIGIT 192 antibodies, and interestingly, two of eight mice rejected tumours after treatment with 193 TIGIT-Fc combined with an anti-asialoGM1 (anti-asGM1) antibody, suggesting that 194 TIGIT-Fc has NK-independent antitumour mechanisms.

195 Notably, the tumour growth effect of TIGIT-Fc, which was significantly weakened in 
196 the $\mathrm{CD}^{+} \mathrm{T}$ cell deficiency context, indicated a unique role of $\mathrm{CD}^{+}$cells in the setting of

197 TIGIT-Fc treatment. To further assess the impact of T cells on the therapeutic effect of

198 TIGIT-Fc, $\mathrm{CD}^{+}$cells or $\mathrm{CD}^{+}$and $\mathrm{CD}^{+} \mathrm{T}$ cells were provided by coimplanting human

199 cells into NSG mice bearing A375 or tumours along with different treatments. Our results

200 demonstrated that reduced tumour growth was only observed in mice treated with

201 TIGIT-Fc together with both $\mathrm{CD}^{+}$and $\mathrm{CD}^{+} \mathrm{T}$ cells, whereas the impact of the TIGIT-Fc

202 therapy with $\mathrm{CD}^{+} \mathrm{T}$ cells alone was minimal (Fig. 4c). Moreover, additional treatment

203 with recombinant CD155 abolished the antitumour effect of TIGIT-Fc, suggesting that

204 the T cell-based antitumour effect of TIGIT-Fc is dependent on CD155. Notably,

205 functional assessment of TIGIT-Fc in vitro in anti-CD3/anti-CD28-stimulated PBMCs

206 from various donors demonstrated the potent ability of this fusion protein to enhance

$207 \mathrm{CD}^{+}$and $\mathrm{CD}^{+} \mathrm{T}$ cell proliferation (Fig. 4d). TIGIT-Fc also significantly increased Th1

208 cytokine production (IFN- $\gamma, \mathrm{TNF}-\alpha$ and IL-2) in these cultures compared with a control

$209 \operatorname{IgG}$ (Fig. 4e).

210 TIGIT-Fc elicits a potent anti-tumour memory response and enhances PD-L1

211 blockade.

212 Our data above showed that TIGIT-Fc has the capacity to evoke potent antitumour

213 immunity involving both $\mathrm{T}$ cells and NK cells. We then tested whether the mice that

214 survived with TIGIT-Fc treatment after tumour challenge have anamnestic responses to

215 tumour re-challenge. MC38 tumour-bearing mice that were initially treated with

216 mTIGIT-Fc and experienced a complete response were subsequently given a second

217 implantation of MC38 cells in the absence of any further treatment (Fig. 5a). Notably, the

218 MC38 rechallenge was incapable of establishing a tumour mass in mice previously cured

219 of tumours, while tumour-naive mice with no previous treatment showed a successful 220 tumour engrafting. Similar results were obtained in CT26 tumour-bearing mice (Fig. 5b)

221 cured with mTIGIT-Fc treatment, where a rechallenge with tumour cells showed no sign

222 of tumour growth in the mice. These results demonstrated that TIGIT-Fc

223 mono-immunotherapy has the capacity to elicit a strong antitumour immune memory.

224 The combination of TIGIT-Fc and atezolizumab that showed a strong antitumour 225 effect in the human T cell-based A375 model is of interest. We therefore investigated 226 whether the combination of mTIGIT-Fc and anti-PD-L1 antibody would enhance the 
227 therapeutic outcome by assessing the potential efficacy of the combined therapy in mice

228 with established syngeneic tumours. In both MC38 and CT26 colorectal adenocarcinoma

229 (Fig. 5c-d), treatment with mTIGIT-Fc or anti-PD-L1 monotherapy resulted in reduced

230 tumour growth, with two of eight and three of eight mice tumour-free after 35 days $231 \quad(25 \%-37.5 \%)$, respectively. Following combinatorial TIGIT-Fc/anti-PD-L1

232 immunotherapy, $75 \%$ and $62.5 \%$ of the MC38- and CT26-inoculated mice, respectively,

233 were tumour-free after 35 days Thus, these data showed that the combined therapy of

234 TIGIT-Fc and anti-PD-L1 induces potent antitumour effects, leading to improved tumour 235 control.

\section{TIGIT-Fc has a potent ADCC effect but no intrinsic effect on tumour cells}

TIGIT-Fc itself may have potent ADCC effect because it is an Fc-fused protein, and 238 therefore we evaluated the ability of TIGIT-Fc to induce ADCC lysis of human tumour 239 cell targets expressing CD155 in vitro utilizing PBMC-dependent ADCC assays. Our data 240 shows that the CD155 high-expressing cell lines A431, SK-BR-3 and SK-OV-3 were 241 sensitive to ADCC-mediated lysis by TIGIT-Fc (Fig. 6a) over a range of E: T ratios,

242 whereas the CD155-negative cell line H2126 was resistant to lysis. We next investigated 243 whether TIGIT-Fc has an intrinsic effect on tumour cells. TIGIT-Fc treatment was 244 evaluated for effects on in vitro cell viability, migration, and invasion in the CD155 245 high-expressing cells. However, no effect on the in vitro proliferation of both SK-BR-3 246 and SK-OV-3 cells was observed after TIGIT-Fc treatment compared with control IgG 247 (Fig. 6b). In migration, invasion and colony formation assays, TIGIT-Fc treatment also 248 showed a negligible effect on tumour cells (Fig. 6c-d). To confirm these findings, the 249 effects of TIGIT-Fc on the in vivo tumour growth of tumour cells were further examined.

250 Tumour growth was monitored in subcutaneous implantation NSG mice models to 251 exclude the impact of immune cells. Our data showed that in the NSG mice, TIGIT-Fc 252 treatment had no detectable effect of tumour growth in both A431 cells and SK-BR-3 253 cells (Fig. 6e). 


\section{Discussion}

TIGIT-Fc was used to simulate membrane-bound TIGIT function in an earlier study ${ }^{9}$.

260 Upon binding with TIGIT-Fc, the activation of CD155 in human monocyte-derived DCs

261 (MDDCs) led to decreased secretion of the proinflammatory cytokine IL-12 and 262 increased secretion of IL-10. It should be noted that in addition to TIGIT-Fc, CD226-Fc 263 also has been shown to modify DC cytokine production, suggesting that this effect is 264 mainly caused by CD155 signalling in MDDCs ${ }^{9}$. Interestingly, subsequent studies 265 reported that agonistic anti-TIGIT mAbs inhibit anti-CD3/anti-CD28 mAb-mediated T 266 cell proliferation and cytokine production in the absence of APCs in humans and mice ${ }^{15-17}$. 267 A recent study further found that the IFN- $\gamma$ production of CD8+ T cells was suppressed 268 by melanoma cells expressing a truncated version of CD155 in a similar manner as cells 269 expressing wild-type $\mathrm{CD} 155^{18}$. Taken together, these studies suggested that the 270 TIGIT-CD155 interaction can inhibit T cell functions without downstream signalling via 271 CD155, highlighting the cell-intrinsic mechanisms of TIGIT. The observation that 272 inhibitory signals can also be directly transmitted via the cytoplasmic tail of TIGIT 273 further supported its cell-intrinsic role. Two publications from the same group established 274 that the ITIM motif is essential for human TIGIT signalling, whereas mouse TIGIT 275 inhibition can be mediated by either the ITIM motif or the ITT motif alone ${ }^{5,19}$. Moreover, 276 another group suggested an important role for the intracellular ITT-like motif in human 277 TIGIT and highlighted two different signalling pathways interfering with NK cell 278 cytotoxicity or IFN- $\gamma$ production ${ }^{20,21}$. Additionally, whole-genome microarray analysis 279 showed that mouse $\mathrm{T}$ cell activation was suppressed by TIGIT engagement by 280 downregulating $\mathrm{T}$ cell receptor (TCR) expression, together with several other molecules 281 involved in TCR and CD28 signalling ${ }^{17}$.

282 Structurally, TIGIT-Fc is an immunoadhesin, which is an immunoglobulin (Ig)-like 283 chimeric protein comprised of target-binding regions fused to the Fc-hinge region of Ig 284 and is designed to have a long half-life and antibody-like properties. Mechanistically, 285 TIGIT-Fc has the capacity to block TIGIT-associated interactions, including the binding 286 of CD155-TIGIT. In a xenograft model system to investigate the T cell-mediated tumour 287 cell killing effect, administration of TIGIT-Fc in A375 xenografts resulted notably in 
tumour growth inhibition. Importantly, the antitumour effect of TIGIT-Fc was entirely

289 dependent upon the presence of the tumour-reactive human T cells in this model. These

290 data unexpectedly demonstrated that the ability of TIGIT-Fc increased tumour cell

291 elimination by $\mathrm{T}$ cells and supported the further investigation of TIGIT-Fc for the

292 treatment of cancer. TIGIT-Fc treatment enhanced NK cell-mediated antitumour

293 immunity both in syngeneic tumour models and tumour xenograft models in nude mice,

294 which is very similar to the effect of an anti-TIGIT antibody ${ }^{14}$, demonstrating that the

295 blockade of TIGIT signalling of tumour-infiltrating NK cells by TIGIT-Fc can be

296 achieved. Interestingly, unlike the anti-TIGIT antibody, which promotes tumour-specific

297 T cell immunity in an NK cell-dependent manner, TIGIT-Fc exhibited a direct activation

298 effect on tumour-specific T cells. We further found that $\mathrm{CD}^{+} \mathrm{T}$ cells were critical for the

299 therapeutic effects of TIGIT-Fc and that TIGIT-Fc treatment augmented proliferation of T

300 cells and mediated Th1 development from naive $\mathrm{CD}^{+} \mathrm{T}$ cells in vitro. These data are

301 consistent with a previous study that showed that agonist anti-CD155 antibodies induced

302 Th1 development in both humans and mice, as evidenced by production of IFN- $\gamma$ and

303 upregulation of $T b \times 21$ transcription ${ }^{22}$. It should be noted that $\mathrm{T}$ cells derived from

304 different healthy donor show different responses to TIGIT-Fc (Fig. 4d-e), indicating a

305 heterogeneity in CD155 signalling-based T cell function.

306 CD155 is a member of the poliovirus receptor-related (PRR) family of adhesion 307 molecules, along with CD111 (nectin-1/PRR-1), CD112 (nectin-2/PRR-2), nectin-3 308 (PRR-3), and nectin-4 (PRR-4). CD155 was initially identified as a receptor for 309 poliovirus in humans ${ }^{23}$ and binds to another PRR family member, nectin-3, as well as to 310 the matrix protein vitronectin, thereby mediating cell-cell or cell-matrix adhesion, 311 respectively, and cell migration ${ }^{24}$. Additionally, CD155 is a ligand for CD226, CD96, and

312 TIGIT on T cells and NK cells. The expression of CD155 in normal tissue, such as 313 epithelial and endothelial cells, is very low, but most tumour cells express high levels of 314 CD155 ${ }^{24}$. Dysregulation of CD155 expression promotes tumour cell invasion, migration, 315 and proliferation and is associated with a poor prognosis and enhanced tumour 316 progression ${ }^{24-26}$, but the definition of tumour cell-intrinsic and -extrinsic effects of CD155 317 has not been fully explored. In present study, we show that targeting CD155 with 318 TIGIT-Fc did not show an effect on the development of tumour cells in vitro, indicating 
319 that at least CD155 did not directly participate in the oncogenic signalling in tumour cells.

320 Moreover, TIGIT-Fc showed a potent ADCC effect, which is well established as a major

321 mode of tumour-cell killing with immune cells, paving the way for TIGIT-Fc as a

322 possible therapeutic candidate for CD155-overexpressing tumours.

323 Our study has several limitations. We provide evidence that promoting 324 tumour-specific immunity with TIGIT-Fc can be achieved, but our in vivo efficacy 325 models may not fully recapitulate human tumours, and the data are from a small number

326 of animals. Moreover, the mechanisms responsible for these therapeutic effects of 327 TIGIT-Fc are currently not well characterized. As some other cells in the tumour 328 microenvironment also express CD155, e.g., tumour-infiltrating myeloid cells, the 329 mechanisms of TIGIT-Fc may be more complicated. Hence, these findings will need 330 further validation.

331 To sum up, our study reveals that TIGIT-Fc can induce the antitumour immune 332 response of NK cells and T cells. Based on the wide application of T cell- and NK 333 cell-based treatment strategies in clinical practice, targeting agents that intervene in the 334 CD155-TIGIT axis hold translational potential. Our results supported the conclusion that 335 treatment with TIGIT-Fc alone or in combination with other immune checkpoint 336 inhibitors is a promising strategy to improve the outcomes of tumour immunotherapy.

Methods

339 Cell lines, primary cells, and therapeutic IgGs

340 The human cell lines A375, A431, SK-BR-3, SK-OV-3, and H2126 were purchased 341 from the American Type Culture Collection (ATCC, Manassas, VA). The identities of the 342 cell lines were verified by STR analysis. CT26 cells were purchased from the Chinese 343 Academy of Sciences (Shanghai, China). MC38 cells purchased from Kerafast, Inc. All 344 cell lines were confirmed to be mycoplasma-free. The cells were maintained in DMEM 345 with $10 \%$ foetal bovine serum. Human peripheral blood mononuclear cells (PBMC) were 346 isolated from healthy volunteer donors by layering over Ficoll-Paque Plus (GE 347 Healthcare) as per the manufacturer's instructions and were enriched for $\mathrm{CD}^{+}$or $\mathrm{CD}^{+} \mathrm{T}$ 348 cells using RosetteSep (STEMCELL Technologies) $\mathrm{T}$ cell enrichment as per the 
manufacturer's instructions. All specimens were collected under an approved protocol by the Second Military Medical University Review Board, and written informed consent was obtained from each donor. The fusion proteins used were as previously described ${ }^{10}$; briefly, a recombinant plasmid was constructed by fusing the Fc segment of human IgG1

353 or murine IG2a, encoding the hinge-CH2-CH3 segment, to the C-termini of the 354 extracellular domains (ECDs) of human and murine TIGIT, respectively. All fusion 355 proteins were obtained via the FreeStyle 293 Expression System (Invitrogen) and 356 subsequently purified using protein A-sepharose from the harvested cell culture 357 supernatant. The purity of the fusion protein was determined by polyacrylamide gel 358 electrophoresis. The protein concentration was measured according to the UV absorbance 359 at a wavelength of $280 \mathrm{~nm}$.

\section{In vivo mice study}

361 Mice were housed in a specific pathogen-free barrier facility. All the in vivo 362 experiments were approved by the Institutional Animal Care and Use Committee 363 (IACUC) of Second Military Medical University. For mouse models with human T cells, 364 tumour-reactive T cells were expanded in culture with mitomycin C-treated cancer cells 365 for 10 days in RPMI 1640 supplemented with 10\% FBS and IL2. Tumour-reactive T cells 366 or subsets were mixed with A375 cells at a 1:5 ratio, and the cell mixtures were implanted 367 by subcutaneous injection of $5 \times 10^{6}$ cells into the flank of NSG mice (Shanghai Model 368 Organisms Center, Inc.). Different drugs were administered i.p. $1 \mathrm{hr}$ immediately after 369 implantation. For syngeneic tumour models, MC38 or CT26 cells were inoculated into $370 \mathrm{C} 57 \mathrm{BL} / 6$ mice or BALB/c mice. For tumour xenograft models, CT26, A431, SK-BR-3 or 371 SK-OV-3 cells were inoculated into BALB/c nude mice (Shanghai Experimental Animal 372 Center of Chinese Academy of Sciences) or NSG mice. When tumour volumes reached 373 an average of approximately $50 \mathrm{~mm}^{3}$, the mice were randomly divided into groups of 8 374 mice each. Multiple dose studies consisted of 3 weeks of treatment, with a $2 \times$ loading 375 dose on the first dose (day of randomization). Tumour volumes were calculated by the 376 following formula: volume $=$ length $\times(\text { width })^{2} / 2$.

\section{Isolation of tumour-infiltrating lymphocytes (TILs)}

378 Tumour tissues were dissociated, and TILs were isolated in the presence of 379 collagenase I $(0.1 \% \mathrm{w} / \mathrm{v}$, Sigma) and DNAse $(0.005 \% \mathrm{w} / \mathrm{v}$, Sigma $)$ for $1 \mathrm{~h}$ before 
380 centrifugation on a discontinuous Percoll gradient (GE Healthcare). Isolated cells were

381 then used in various cell function assays

382 Flow cytometry

383 Cell surface staining was performed at $4{ }^{\circ} \mathrm{C}$ for $30 \mathrm{~min}$ and was analysed using a

384 FACSCalibur flow cytometer (BD Biosciences) and CellQuest Software (BD

385 Biosciences). A minimum of $1 \times 10^{4}$ cells was examined. Antibodies to mouse CD3 $\varepsilon$

386 (145-2C11, BD Bioscience), CD107a (1D4B, BD Bioscience), IFN- $\gamma$ (XMG1.2, 387 BioLegend), TNF (MP6-XT22, BioLegend), CD155 (4.24.1, BioLegend), CD8 $\alpha$ (53-6.7,

388 BioLegend) and antibodies to human CD3 (HIT3a, BioLegend), CD45 (2D1, BioLegend),

389 CD4 (RPA-T4, BD Bioscience), and CD8 (RPA-T8, BD Bioscience) were used in this 390 study. For TILs experiments, the cells were stimulated with $30 \mathrm{ng} / \mathrm{ml}$ phorbol 391 12-myristate 13-acetate (PMA, Sigma) and $1 \mu \mathrm{M}$ ionomycin (Sigma) in the presence of

$3922.5 \mu \mathrm{g} / \mathrm{ml}$ monensin (eBioscience) for $4 \mathrm{~h}$, after which they were stained for surface

393 markers and fixed and permeabilized with eBioscience FoxP3 fixation buffer according 394 to the manufacturer's instructions. Fixed cells were stained with antibodies to IFN- $\gamma$ 395 (XMG1.2; BioLegend) and TNF (MP6-XT22, BioLegend).

\section{In vitro ADCC assay}

Target cells were labelled with ${ }^{51} \mathrm{Cr}$ for $1 \mathrm{~h}$ at $37{ }^{\circ} \mathrm{C}$. Radioactive ${ }^{51} \mathrm{Cr}(50 \mu \mathrm{Ci})$ was used to label $1 \times 10^{6}$ target cells. Labelled target cells $(n=5000)$ were plated in each well of a 96-well plate in a volume of one hundred microliters. Effector cells were added at a volume of $100 \mu \mathrm{l}$ at different E:T ratios with different drugs. The cells were incubated together for $4 \mathrm{~h}$ at $37{ }^{\circ} \mathrm{C}$. The supernatant $(30 \mu \mathrm{l})$ from each well was collected and

403 Radioactivity released into the culture medium was measured using a $\beta$-emission-reading 404 liquid scintillation counter. The percentage of specific lysis was calculated as follows: 405 (sample counts - spontaneous counts) $/($ maximum counts - spontaneous counts) $\times 100$.

\section{In vitro tumour cell line assay}

407 For cell viability assay, tumour cells were plated in triplicate at $5 \times 10^{3}$ per well in 408 96-well plates overnight. After plating, the cell culture medium was replaced with assay 409 medium containing $0.1 \%$ FBS. TIGIT-Fc and control IgG were added at multiple 410 concentrations, and 72 hours later, cell viability was measured using the CellTiter-Glo 
411 Luminescent Cell Viability Assay (Promega). For the migration assay, cell mobility was

412 assessed with a modified two-chamber migration assay (8-mm pore size, BD Biosciences,

413 Bedford, MA) according to the manufacturer's instructions as described previously ${ }^{27}$

414 Briefly, approximately $2 \times 10^{4}$ cells were plated into the upper chamber for 18-24 hours

415 along with different treatments $(1 \mathrm{mg} / \mathrm{ml})$ and allowed to migrate into the lower chamber.

416 The cells at the bottom of the membrane were fixed and stained with $20 \%$ methanol/0.2\%

417 crystal violet, while the cells in the upper chamber were removed using cotton swabs.

418 The invasion assay was performed as previously described using transwell cell culture

419 chambers $(8-\mu \mathrm{m} \text { pore size polycarbonate membrane, Costar })^{28}$. Briefly, $100 \mu$ of cell

420 suspension at $1 \times 10^{6}$ cells $/ \mathrm{ml}$ with different treatments $(1 \mathrm{mg} / \mathrm{ml})$ in DMEM

421 supplemented with $0.5 \%$ foetal bovine serum was loaded into the upper chamber, and the

422 lower chamber was loaded with $600 \mu$ of DMEM with $10 \%$ foetal bovine serum. The

423 membranes were precoated with Matrigel (BD Pharmingen). The chamber plates were

424 incubated at $37^{\circ} \mathrm{C}$ for 24 hours. Then, the filter was fixed in $4 \%$ paraformaldehyde and

425 stained with haematoxylin (Sigma). The cells on the upper side of the filter were removed

426 with a cotton swab, and the cells that had passed through the membrane were counted in

42710 randomly selected microscopic fields. For the colony formation assay, the cells were

428 seeded onto 6 -well plates or $3.5-\mathrm{cm}$ dishes along with different treatments $(1 \mathrm{mg} / \mathrm{ml})$.

429 Colonies were allowed to form in an incubator at $37^{\circ} \mathrm{C}$ and $5 \% \mathrm{CO}_{2}$ for 10 days. At the

430 end of the incubation period, the clones were fixed and stained with $0.5 \%$ crystal violet,

431 and colonies larger than $50 \mu \mathrm{m}$ in diameter were counted.

\section{Statistical analysis}

433 Unless otherwise specified, the Student's t-test was used to evaluate the significance 434 of differences between 2 groups, and ANOVA was used to evaluate differences among 3 435 or more groups. Differences between samples were considered significant when $P<0.05$.

\section{Conflicts of interest}

437 The authors declare the following competing interests: J.Z. is a shareholder at 438 KOCHKOR Biotech, Inc., Shanghai; W.F. and S.H. are inventors on intellectual property 439 related to this work. No potential conflicts of interest were disclosed by the other authors. 


\section{Acknowledgements}

This study was supported by the National Natural Science Foundation of China

443 (grant nos. 81773261, 31970882, 81903140 and 81602690); the Shanghai Rising-Star

444 Program (grant no. 19QA1411400); the Shanghai Sailing Program (19YF1438600); and

445 the Shanghai Chenguang Program (grant no. 17CG35).

\section{$446 \quad$ Figure Legends}

447 Figure 1. TIGIT-Fc shows antitumour activity in xenograft mouse models of human

448 cancer. a. The binding of atezolizumab and TIGIT-Fc to A375 cells was detected by flow

449 cytometry analysis. The histograms shown in black correspond to the isotype controls,

450 whereas the red histograms indicate the positive fluorescence. b-c. Tumour volumes of

451 A375 in NOD/SCID mice following coimplantation of primary human $\mathrm{T}$ cells for

452 assessing the therapeutic effect of atezolizumab in combination with TIGIT-Fc (b) or an

453 anti-TIGIT antibody (c). Antibodies were used in a dose of $10 \mathrm{mg} / \mathrm{kg}$ alone or in

454 combination ( $5 \mathrm{mg} / \mathrm{kg}$ each), as specified in the figure. The data are the means \pm s.d., $n=$

455 8. $P$ values were generated by two-way ANOVA followed by a Bonferroni posttest

456 comparison.

458 Figure 2. TIGIT-Fc inhibits tumour growth and prevents exhaustion of

459 tumour-infiltrating NK cells. a. Tumour volumes of different MC38 tumour xenografts

460 after the indicated weekly treatment with mTIGIT-Fc $(10 \mathrm{mg} / \mathrm{kg})$ or control $\mathrm{IgG}$, as

461 specified in the figure. b. Survival of mice as in a at various times. $P$ values, Mantel-Cox

462 test. c-d. Frequency of cells expressing CD107a, TNF, IFN- $\gamma$ or CD226 among

463 tumour-infiltrating NK cells (c) or T cells (d) in mice as in $\mathbf{a}$ ( $\mathrm{n}=6-8$ per group), assessed

464 when the tumours in the mice treated with mTIGIT-Fc reached a size of $\sim 250 \mathrm{~mm}^{3}$. e.

465 Tumour volumes of different CT26 tumour xenografts after the indicated weekly

466 treatment with mTIGIT-Fc $(10 \mathrm{mg} / \mathrm{kg})$ or control $\mathrm{IgG}$, as specified in the figure. f.

467 Survival of mice as in e at various times. $P$ values, Mantel-Cox test. $\mathbf{g}$-h. Frequency of

468 cells expressing CD107a, TNF, IFN- $\gamma$ or CD226 among tumour-infiltrating NK cells (g)

469 or T cells (h) in mice as in $\mathbf{e}(\mathrm{n}=6-8$ per group). 
471 Figure 3. TIGIT has a protective role in mice with adaptive immunodeficiency. a

472 Tumour volumes of different CT26 tumour xenografts in nude mice after the indicated 473 weekly treatment with mTIGIT-Fc $(10 \mathrm{mg} / \mathrm{kg})$ or control $\mathrm{IgG}$, as specified in the figure. b. 474 Frequency of cells expressing CD107a, TNF, or IFN- $\gamma$ among tumour-infiltrating NK 475 cells in mice as in a ( $\mathrm{n}=6-8$ per group), assessed when tumours in mice treated with 476 mTIGIT-Fc reached a size of $\sim 250 \mathrm{~mm}^{3}$. c. Tumour volumes of different A431, SK-BR-3, 477 SK-OV-3, H2126 or H2126-CD155 tumour xenografts in nude mice after the indicated 478 weekly treatment with TIGIT-Fc $(10 \mathrm{mg} / \mathrm{kg})$ or control IgG, as specified in the figure. d. 479 The expression of CD155 in different tumour cell lines was detected by staining with an 480 anti-CD155 antibody, followed by flow cytometry analysis. The histograms shown in 481 black correspond to the isotype controls, whereas the red histograms indicate the positive 482 fluorescence.

Figure 4. CD4+ T cells contribute to the antitumour efficacy of TIGIT-Fc. a. Tumour volumes of different CT26 tumour xenografts after the indicated weekly treatment with mTIGIT-Fc $(10 \mathrm{mg} / \mathrm{kg})$ or control IgG, or plus depletion of different immune cells as 487 specified in the figure: anti-CD8 (53.5.8, $\mathrm{CD}^{+} \mathrm{T}$ cell depletion); anti-CD4 (GK1.5, CD4 ${ }^{+}$ 488 T cell depletion), anti-asGM1 (NK cell depletion) (antibodies were given weekly at the 489 dose of $10 \mathrm{mg} / \mathrm{kg}$ ). b. Survival of mice in a. c. Tumour volumes of A375 in NSG mice 490 following coimplantation of different primary human T cells for assessing the therapeutic 491 effect of TIGIT-Fc. d-e. proliferation assay by CFSE dilution (d) and ELISAs for IL-2, 492 IFN- $\gamma$, and TNF production (e) after stimulation of PMBCs with plate-bound 493 anti-CD3/CD28 plus TIGIT-Fc or control IgG.

495 Figure 5. TIGIT-Fc induces an antitumour memory response and shows a synergic 496 effect with anti-PD-L1. a-b. Tumour rechallenge experiments. The surviving mice xenografted with MC38 (a) or CT26 (b) after mTIGIT-Fc treatment were rechallenged

498 with different tumours. c-d. Tumour volumes of different MC38 (c) or CT26 (d) tumour 499 xenografts after the indicated weekly treatment with mTIGIT-Fc $(10 \mathrm{mg} / \mathrm{kg})$ or control $500 \mathrm{IgG}$, as specified in the figure. 


\section{Figure 6. TIGIT-Fc shows an ADCC effect but no intrinsic effect on tumour cells. A.}

503 In vitro ADCC assay using tumour cell lines with different levels of CD155 expression as

504 targets. b. The effects of TIGIT-Fc on the in vitro viability of different tumour cells. c-d.

505 The effects of TIGIT-Fc on migration, invasion and colony formation of SK-BR-3 (c) and

506 SK-OV-3 cells (d). e. Tumour volumes of different SK-BR-3 or SK-OV-3 tumour 507 xenografts in NSG mice after the indicated treatment. 
Figure 1

a

A375
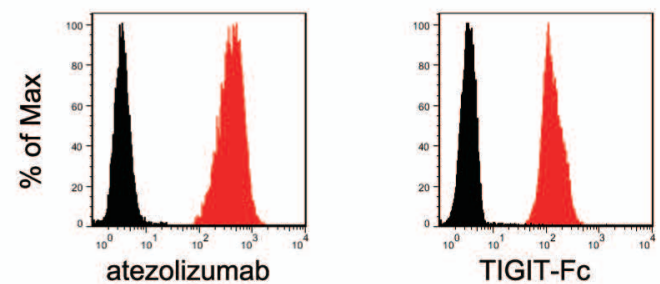

b

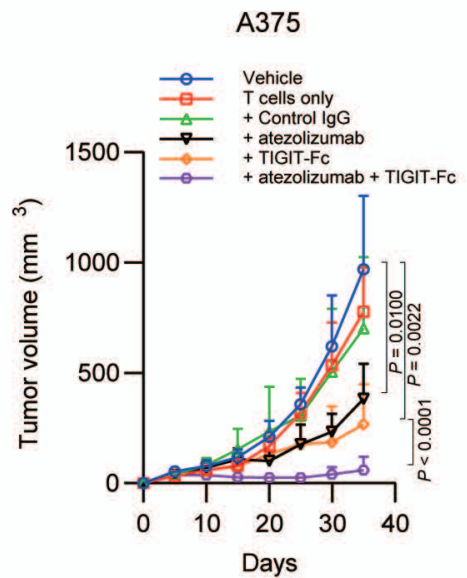

c

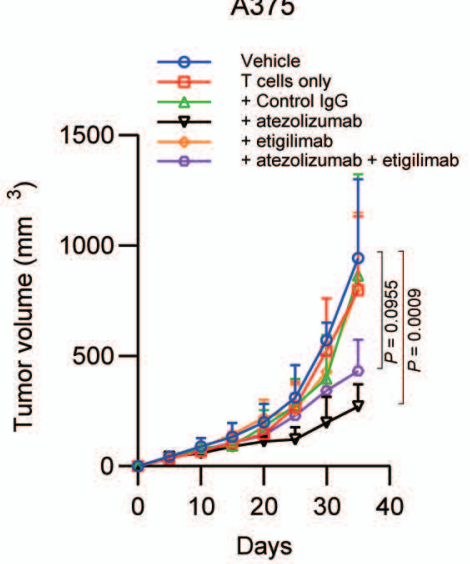




\section{Figure 2}

a

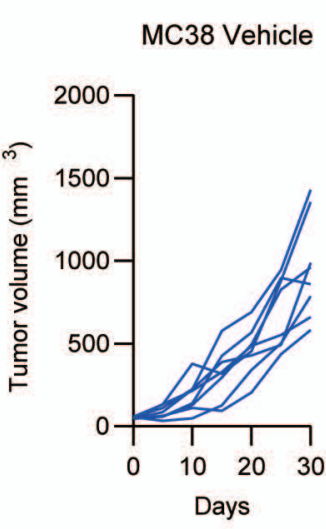

Days

e

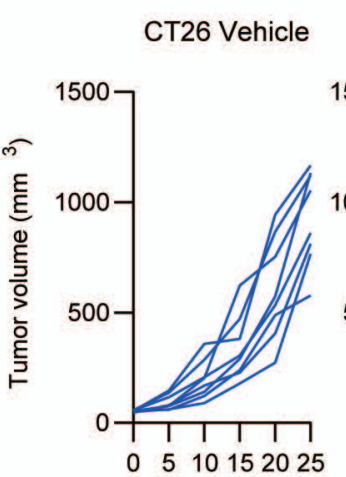

Days

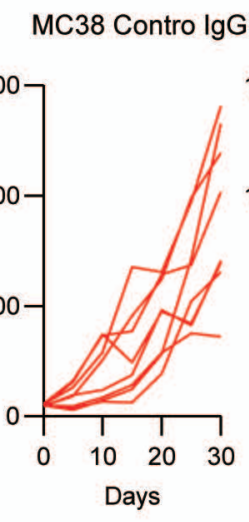

Days b

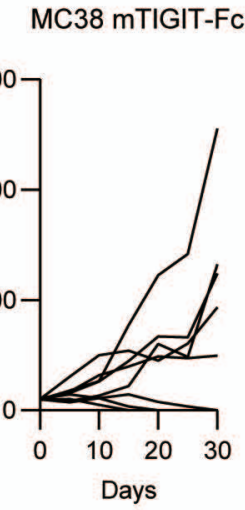

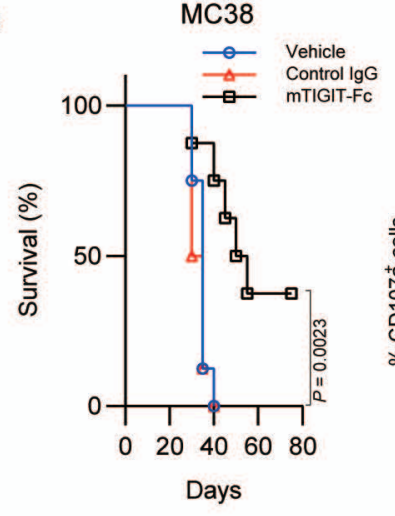

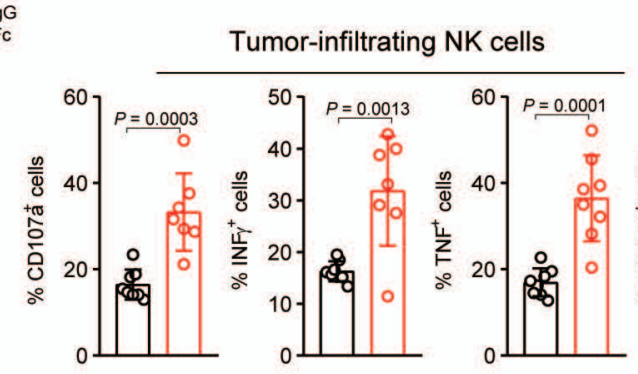

umor-infiltrating NK cells

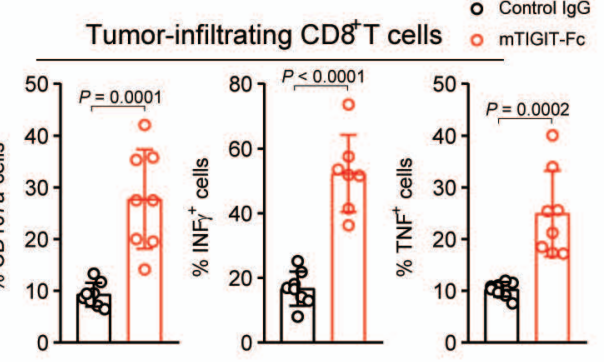

g

h
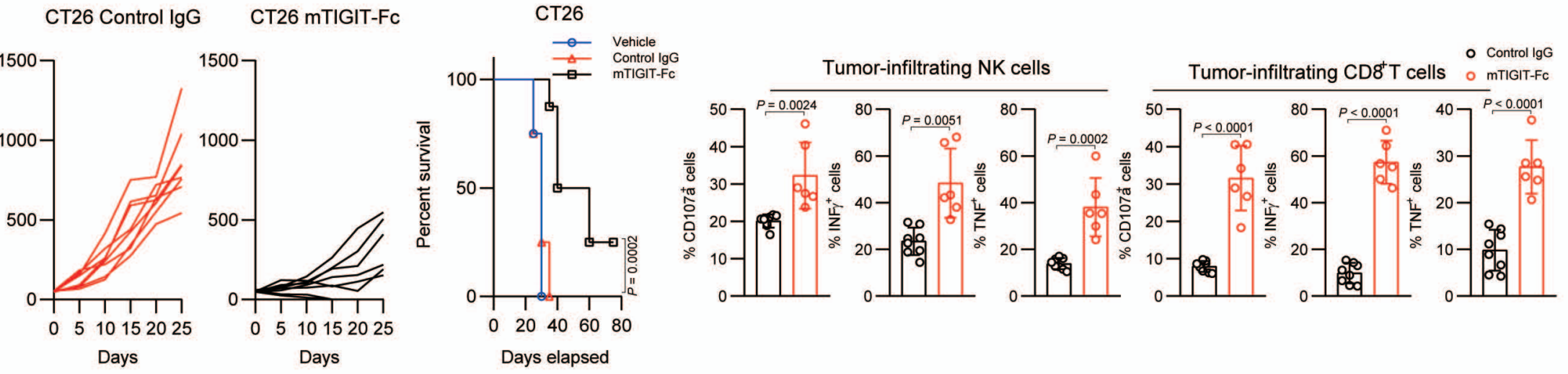
Figure 3

a

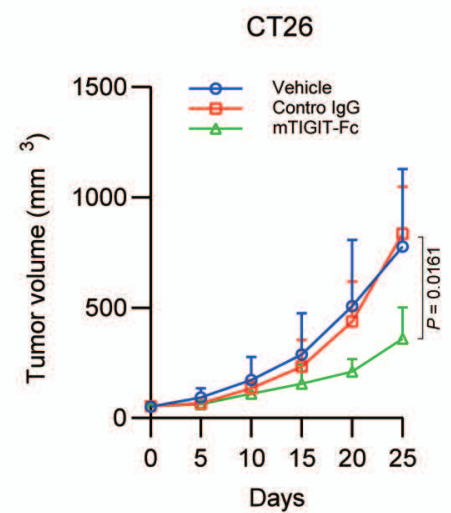

b

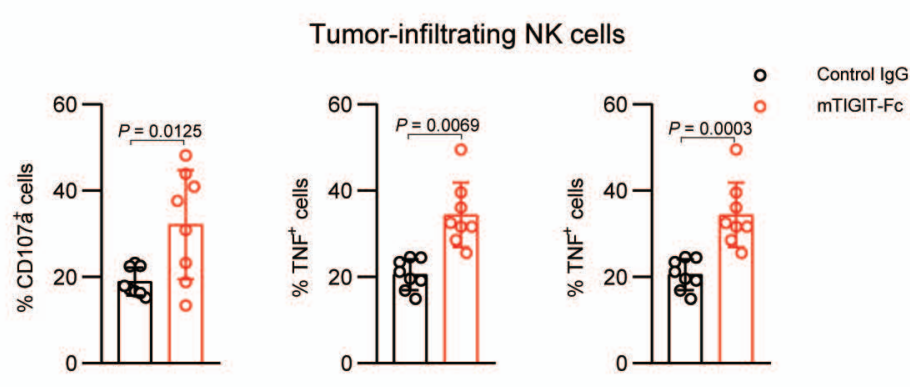

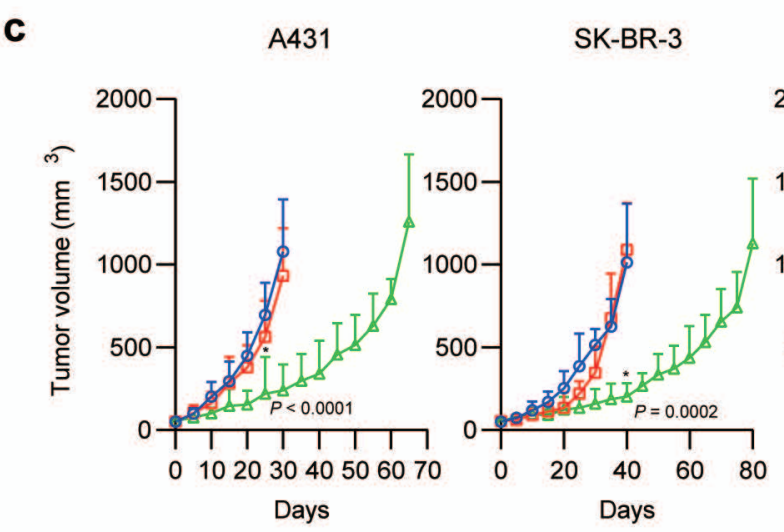
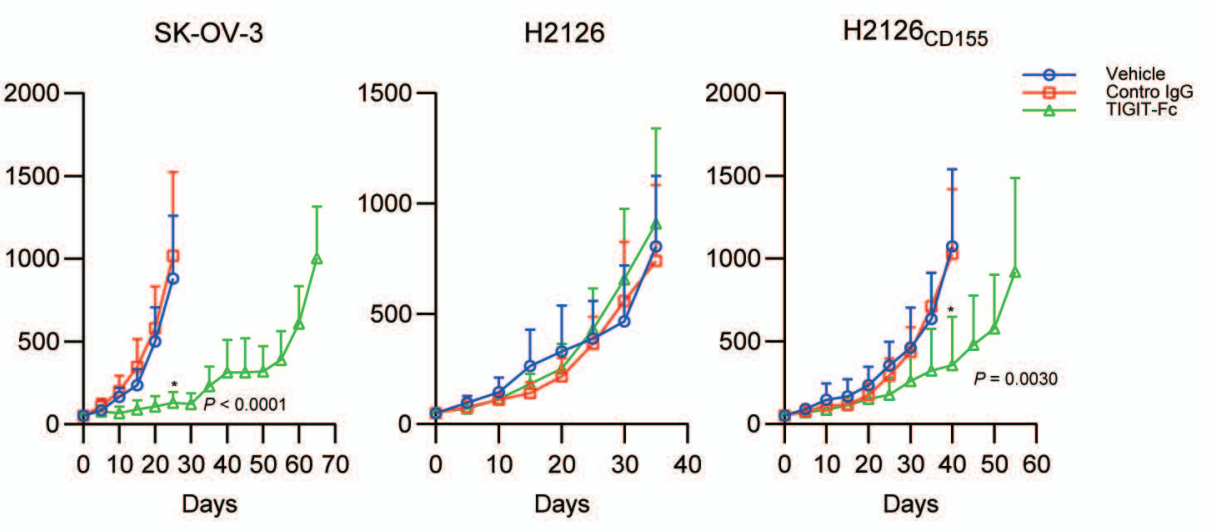

d
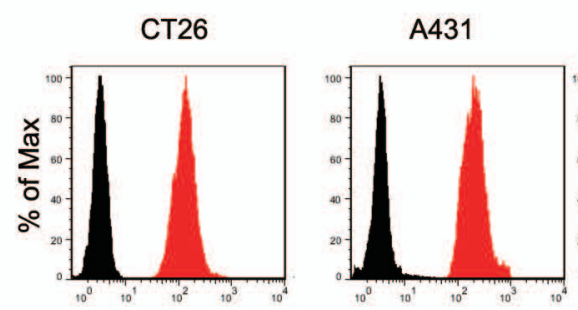

SK-BR-3
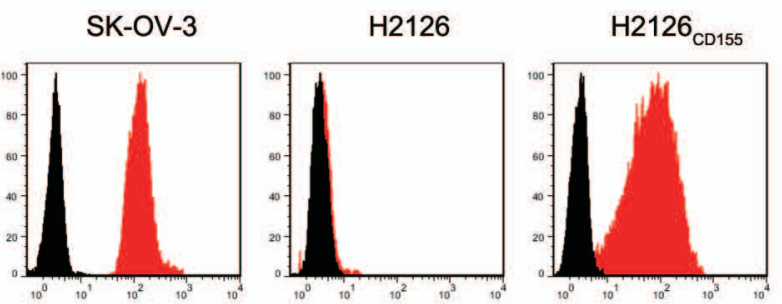

CD155 


\section{Figure 4}
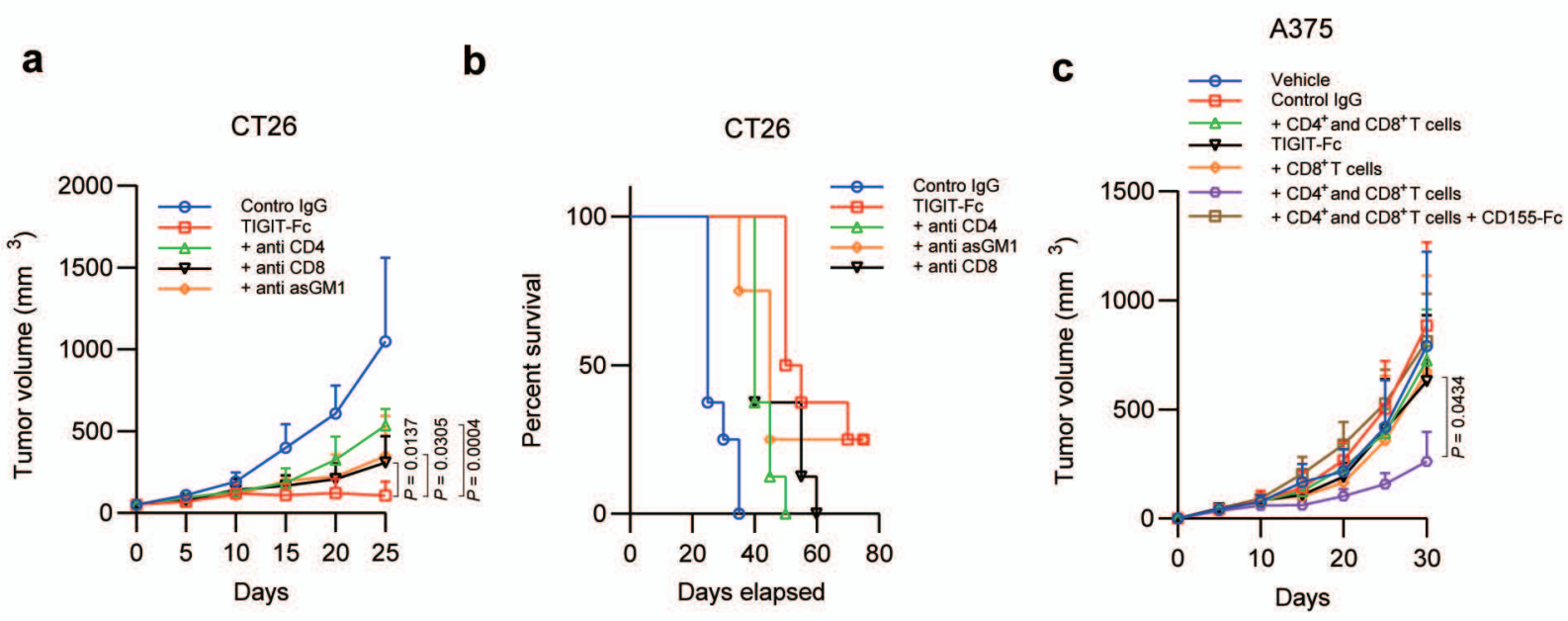

d

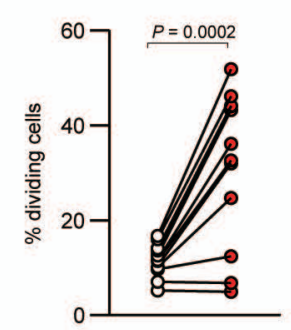

e

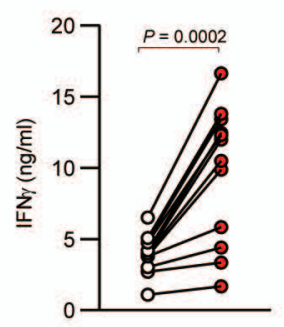

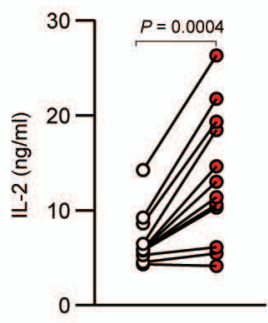

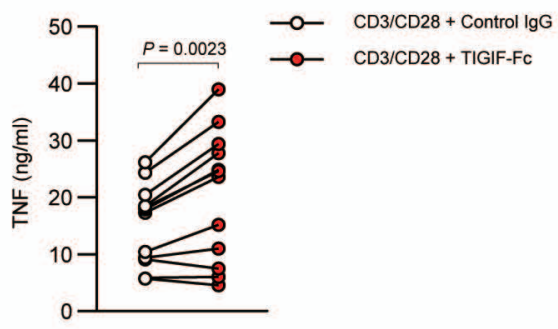


Figure 5

a

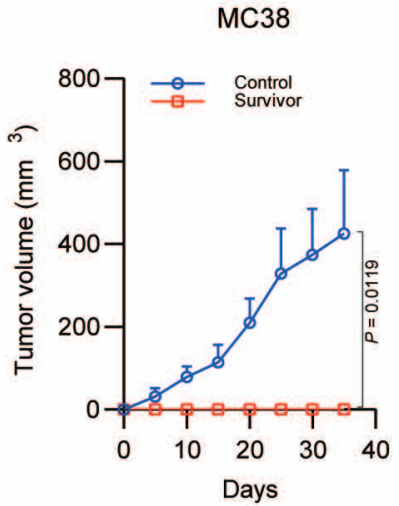

C

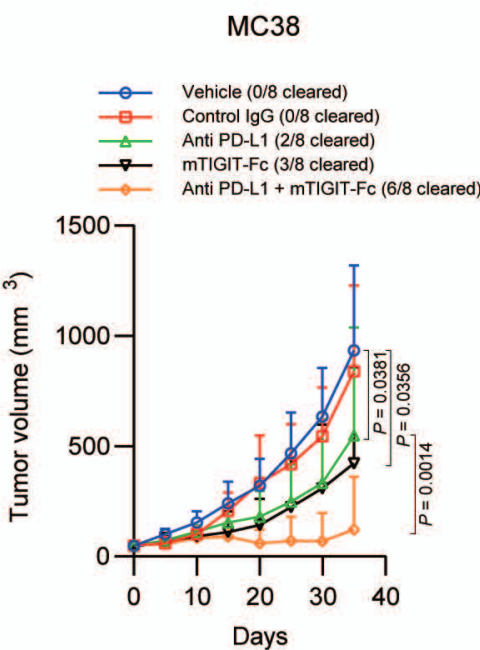

b

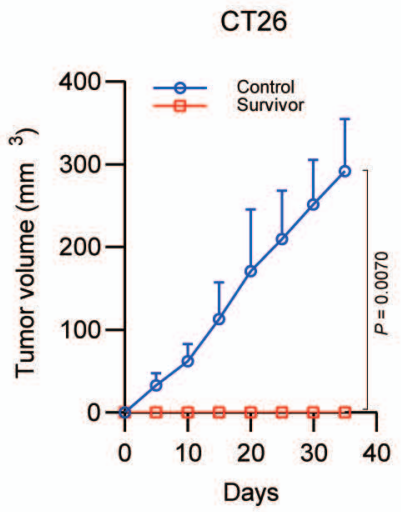

d

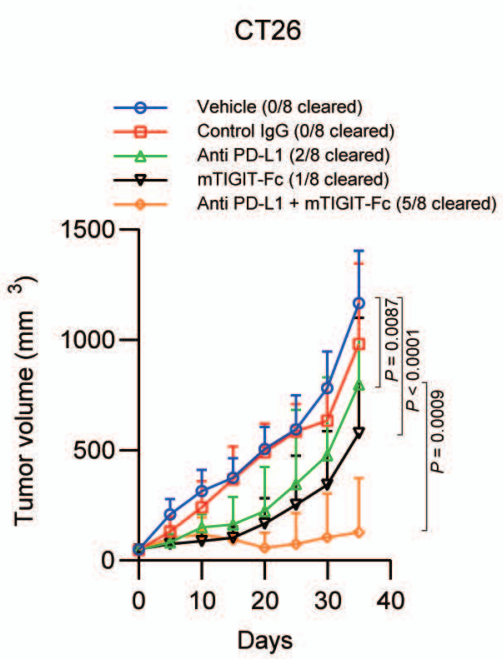




\section{Figure 6}

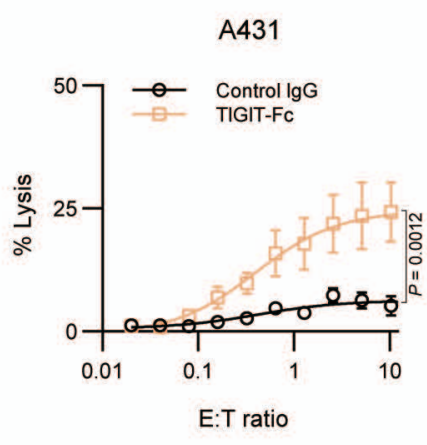

b

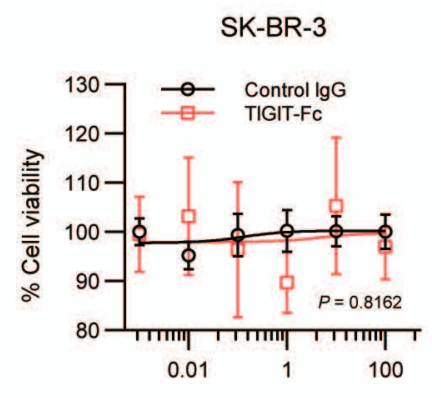

d

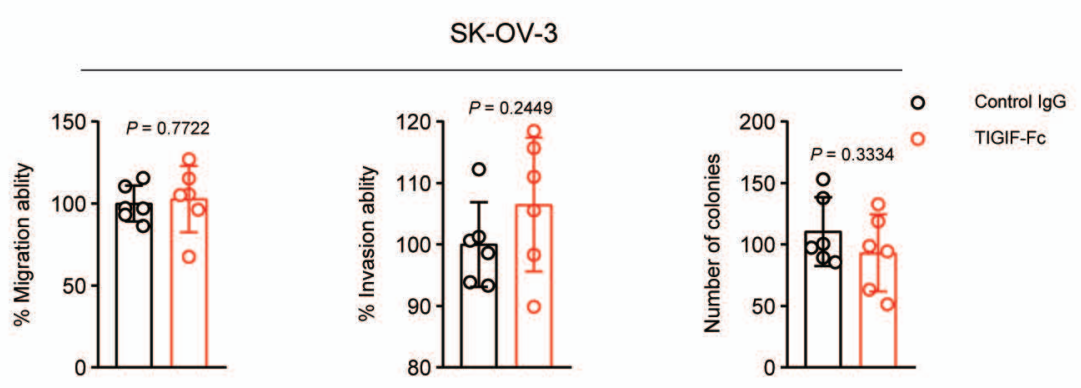

c

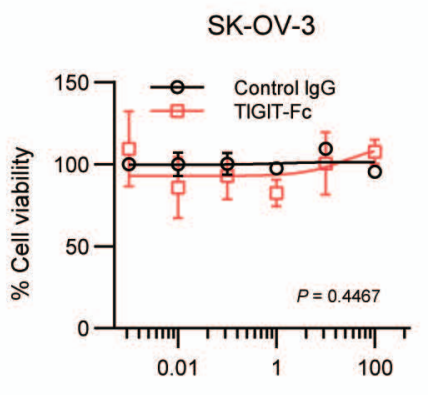

Drug concentration (ng/ml)
H2126
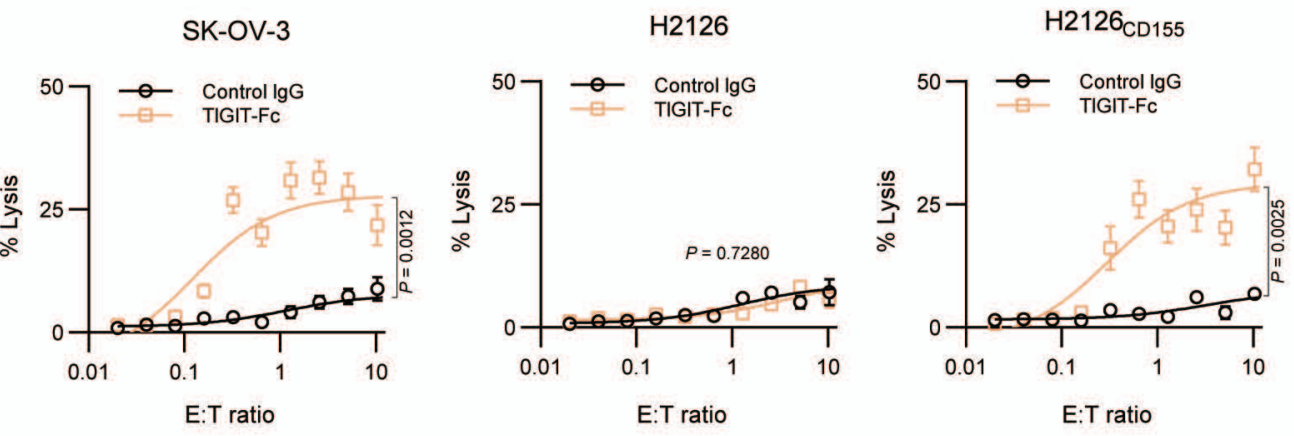

SK-BR-3
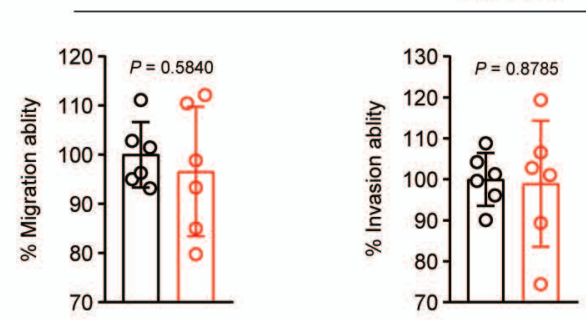

- Control $\lg G$
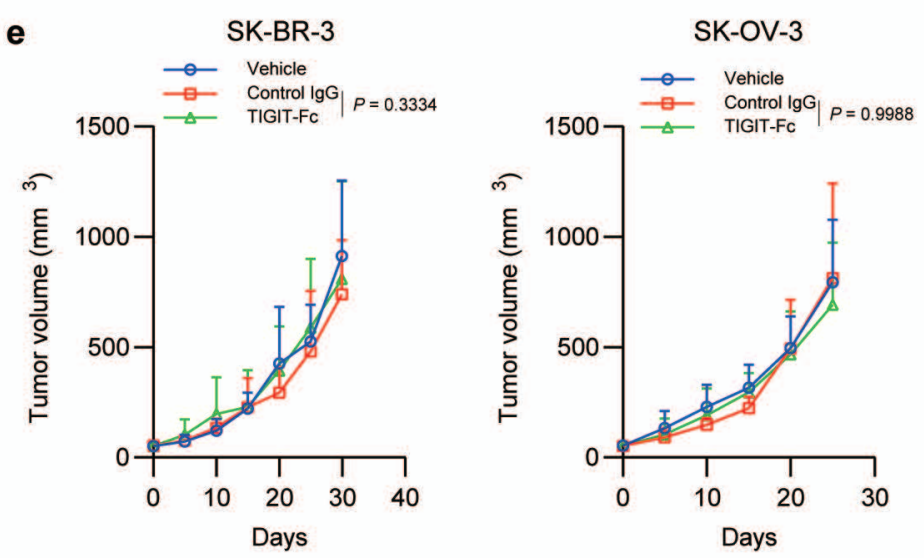